\title{
Retinal Fundus Image Research for Diagnosis of Diabetic Maculopathy
}

\author{
Joshua Thomas, K.S. Angel Viji
}

\begin{abstract}
Fundus images are valuable resources in diagnosis of retinal diseases. This paper proposes a computer-aided method based on various feature extraction techniques and support vector machines (SVM) for detection and classification of diabetic maculopathy (DM). DM, defined as retinopathy within one disc diameter of the centre of the macula, is a major cause of sight loss in diabetes. Here, we bring out a new approach to detect DM based on retinal fundus image features. During the first stage the input image is enhanced and the optic disc is masked to determine the presence of regions of foveal neighborhood. The second stage, deals with various feature extraction technique based on transform, shape and texture features. Extracted features are further categorized as healthy or affected images. Here we go for classification task using the RBF Support Vector Machine (SVM) classification, the techniques have been tested on retinal databases and these are compared with trained phase to categorize Healthy and DM images. This method can detect DM with a level accuracy on par with human retinal specialists.
\end{abstract}

\section{INTRODUCTION}

Diabetes is a chronic disease that occurs when the pancreas is no longer able to make insulin, or when the body cannot make good use of the insulin it produces [1]. Over time, diabetes can damage the heart, blood vessels, eyes, kidneys, nerves and blood vessels (BV) of the retina [2]. The metabolic variations occurring in retinal BVs because of abnormal blood flow and the medical attributes get stored over and over the macula, causing swelling of the macular area is called as diabetic maculopathy (DM). It is a condition that can result from diabetic retinopathy and a main reason of visual impairment. It happens when diabetes harms the little veins in the macula, the light-touchy tissue behind the eye [3]. A healthy macula is important for good vision.

The only way to control this disease that affects and threatens the eyesight is through early detection and welltimed treatment. Techniques of extracting important information from images captured in a constrained environment are being studied for several decades to develop images or to make automated systems. But in the recent years, increased demand of intelligent automated systems necessitated the processing of more challenging real world scenes which possess many complexities; like noise in data, abrupt motion or illumination variation, non-rigid or articulated movement of objects, background variation etc. With this objective in this paper, an automatic method for detection and analysis of the DM in color fundus image is being presented.

The preprocessing is mainly on Optic Disc (OD)

\footnotetext{
Manuscript received September 16, 2019.

Joshua Thomas, Research Scholar, Department of Electronics and Communication Engineering, Noorul Islam University, Tamil Nadu, India. (e-mail: joshuaalackal@gmail.com).

K.S. Angel Viji, Research Supervisor, Department of Computer Science Engineering, Noorul Islam University, Tamil Nadu, India.(e-mail:
} ksangelviji@gmail.com) detection and masking, based on the group of features extracted from retinal fundus images like Green channel separation, Contrast enhancement, canny edge detection, Morphological opening, closing etc [4]. The OD region contains maximum BVs whereas, macula and fovea is devoid of BV. Detected OD is then masked by separating the extracted image from morphologically processed image; the location of macula plane is identified by intensity variations of affected pixels. When the input data is too large to be processed and suspected to be redundant then the data is transformed into a reduced set of feature representations. The process of transforming the input data into a set of features is called feature extraction. Features often contain information relative to shape, frequency, texture or context. In image classification, the local features of the image are utilized to distinguish the different images.

Applying a combination of these features, the proposed method separates Healthy/DM Images using the Support Vector Machine (SVM) classification. SVM is the most commonly used classification algorithm for disease prediction. The SVM classifier builds a model to predict classes for new examples [5]. It assigns new example/data points to one of the classes with associated learning algorithms and analyzes data used for classification. These classes can be termed as the training phase since we are training the image as whether it contains the maculopathy texture or not. The presence of lesions in these regions is compared with trained phase to diagnose and categorize Healthy and DM images.

\section{DIABETIC AND RETINAL FAILURE}

The term diabetes describes a metabolic disease with disturbances of carbohydrate, fats and protein, because of defects in insulin secretion, insulin movement, or every. The long-term results of diabetes include modern development of the particular headaches of retinopathy with potential blindness, nephropathy that could cause renal failure, and/or neuropathy with chance of foot ulcers, amputation, and functions of autonomic dysfunction, along with sexual dysfunction [4]. People with diabetes are at multiplied threat of cardiovascular, peripheral vascular and cerebrovascular disorder [5].

Diabetic retinopathy (damage to the retina) is caused by complications of diabetes, which can eventually lead to blindness [6]. In this stage, tiny blood vessels within the retina leak blood or fluid. The leaking fluid causes the retina to swell or to form deposits. Non-proliferative diabetic retinopathy (NPDR), commonly known as background 
retinopathy is an early stage of diabetic retinopathy. In this stage, tiny blood vessels within the retina leak blood or fluid. The leaking fluid causes the retina to swell or to form deposits called exudates [7]. Many people with diabetes have mild NPDR, which usually does not affect their vision. When vision is affected, it is the result of macular edema and/or macular ischemia. Diabetic Retinopathy (DR) is the result of microvascular retinal changes triggered by diabetes and it is the most common leading cause of preventable blindness in the working-age population in the world $[8,9]$. Whereas, Diabetic Macular Edema (DME) is a complication associated with DR, Data characterized by accumulation of fluid or retinal thickening that can occur at any stage of DR $[10,11]$. Macular edema is swelling, or thickening, of the macula, a small area in the center of the retina that allows us to see fine details clearly. The swelling is caused by fluid leaking from retinal blood vessels. It is the most common cause of visual loss in diabetes. Prolonged diabetes causes severe damage to the vision through leakage of blood and blood constituents over the retina [8]. The effect of the leakage becomes more threatening when these abnormalities involve the macula. This condition is known as diabetic maculopathy and it leads to blindness, if not treated in time.

\section{PROPOSED METHODOLOGY \& RESULTS}

Here we open up on Digital Image Processing (DIP) to develop computer algorithm in retinal fundus image to extract and detect useful information of diabetic maculopathy. The detailed methodology and process of the proposed DM detection is been described in the below block diagram [Fig.1]. The environment used for programming is MATLAB 2018 based on a processor of core i5.

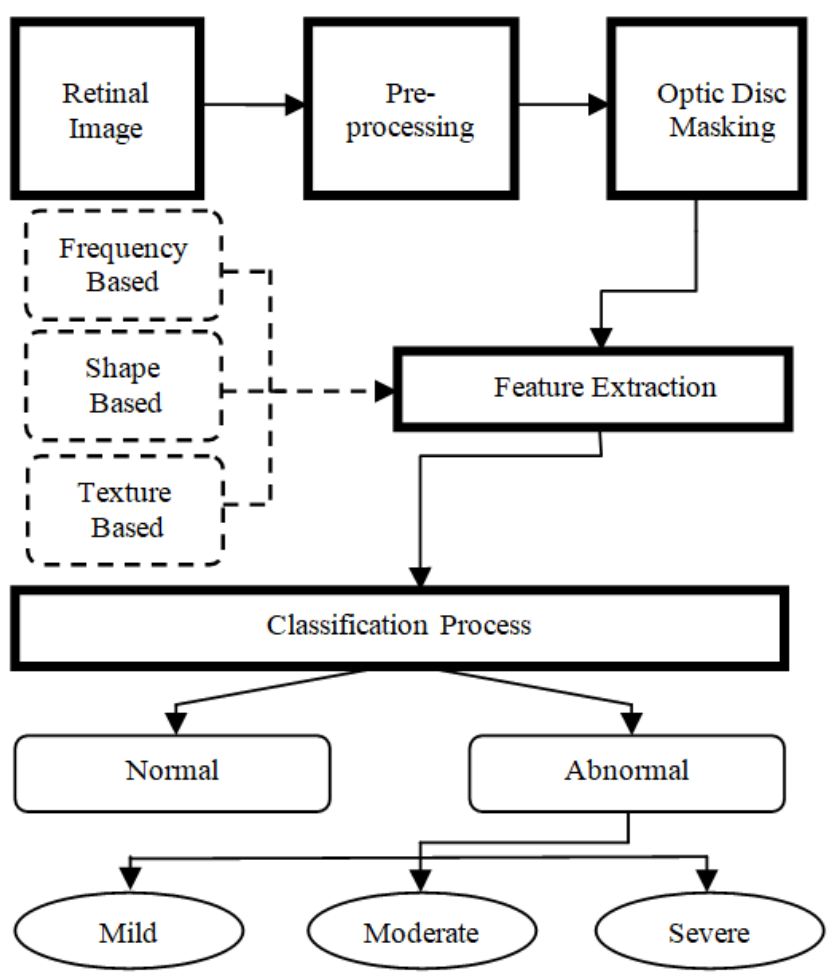

Fig. 1: Flow Diagram of Proposed System

The method consists of 1) the enhancement of the input image and the optic disc masking and 2) the transforming the input image into a set of the extracted features want to be classified as belonging to Healthy or affected images.
Fig. 1 shows the flow diagram of proposed system. For evaluating the proposed algorithm, three publicly available fundus image databases are used and evaluate the images in real time.

In order to identify the maculopathy, the localization and the detection of macula and fovea are essential, as the maculopathy is represented by lesions in the macula region and fovea is the centre of macula. The optic disc identification, masking and blood vessels extraction are performed prior to the detection of the macula by using the optic disc uncovering and masking process, followed by various feature extraction techniques and SVM for detection and classification of diabetic maculopathy (DM).

The rest of this paper is arranged as follows. Section $A$ describes Physiology of Retina, Section $B$ image databases and Preprocessing, Section $C$ Optic disc detection and masking, while Section $D$ is the Detection and analysis of diabetic maculopathy, Section $E$ extracted features and Section $F$ SVM classifier along with the experimental results and discussion, followed by concluding remarks.

\section{Eye Anatomy Facts}

The eye is often compared to a camera. It gathers light and then transforms that light into a "picture." The lenses focus the incoming light. Just as a camera focuses light onto the film to create a picture, the eye focuses light onto a specialized layer of cells, called the retina, to produce an image. The retina [Fig. 2] is the third and inner coat of the eye which is a light-sensitive layer of tissue. The optics of the eye create an image of the visual world on the retina (through the cornea and lens), which serves much the same function as the film in a camera [9]. Light striking the retina initiates a cascade of chemical and electrical events that ultimately trigger nerve impulses. These are sent to various visual centres of the brain through the fibres of the optic nerve.

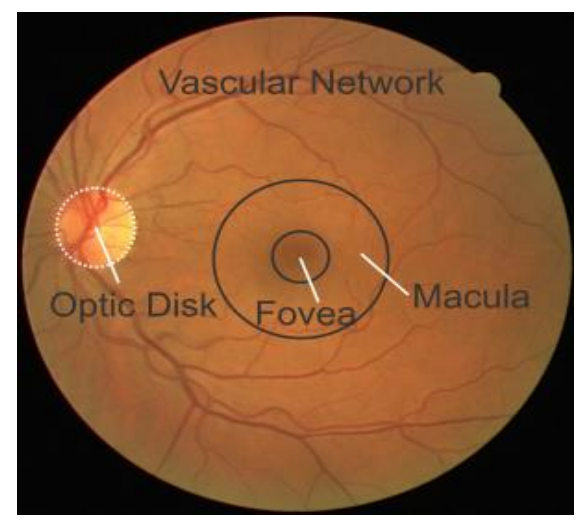

Fig. 2: Fundus Image with its Main Features

The retina acts like the film in a camera to create an image. When focused light strikes the retina, chemical reactions occur within specialized layers of cells. These chemical reactions cause electrical signals, which are transmitted through nerve cells into the optic nerve, which carries these signals to the brain, where the electrical signals are converted into recognizable images. There are four main 
parts in retina based on analysis of diabetic maculopathy are explained as follows [9].

\section{- Vascular Network and Optic Disc}

The vascular network projects from the retina into the the transparent jelly-like tissue filling the eyeball; it supplies oxygen and nutrients to the eye, and may also aid in vision. The retina requires a constant supply of blood to make sure your cells get enough nutrients and oxygen. Blood also removes the waste your retina produces. The human retinal vascular imaging, as a noninvasive research tool, can provide precise quantification of fine retinal vascular changes associated with retinal vascular diseases [10].

The optic disc is the point of exit for ganglion cell axons leaving the eye. Because there are no rods or cones overlying the optic disc, it corresponds to a small physiological blind spot in each eye. The optic disc represents the beginning of the optic nerve and is the point where the axons of retinal ganglion cells come together.

The optic disc is also the entry point for the major blood vessels that supply the retina. The optic disc in a normal human eye carries from 1 to 1.2 million neurons from the eye towards the brain. The optic disc is placed 3 to $4 \mathrm{~mm}$ to the nasal side of the fovea. It is a vertical oval, with average dimensions of $1.76 \mathrm{~mm}$ horizontally by $1.92 \mathrm{~mm}$ vertically $[9,10]$.

\section{Macula and Fovea}

The macula is located in the central part of the retina and has the highest concentration of cones. It is the area of the retina that is responsible for providing sharp central vision. The macula is an oval-shaped pigmented area near the center of the retina of the human eye. It has a diameter of around $5.5 \mathrm{~mm}$.

The clinical macula is seen when viewed from the pupil, as in retinal photography. Near its center is the fovea, a small pit that contains the largest concentration of cone cells in the eye and is responsible for central, high-resolution vision. The umbo is the center of the fovea which is located at the centre of fovea [9, 10 and 11].

\section{Image Database and Pre-processing}

In this work, the input fundus images used are obtained from the DRIVE (Digital Retinal Images for Vessel Extraction) [12], MESSIDOR (Methods to evaluate segmentation and indexing techniques in the field of retinal ophthalmology) [14] and HRF (High-Resolution Fundus Image) [15] dataset. Fig. 3 shows fundus images of DRIVE and MESSIDOR databases. These datasets provide information on the disease severity of diabetic retinopathy, and diabetic macular edema for each image.

This section deals mostly with traditional methods of image Pre-processing. The uneven illumination increases the intensity level near OD and decreases the intensity towards the regions away from OD. This severely affects the illumination of lesions and macula.

Thus, for reducing the intra and inter image variability, pre-processing step is very much significant for better performance of the algorithm. The fundus image databases used in this work have resolution and illumination variations. To reduce its impact on the algorithm, all the images are resized to $350 \times 350$ for making the evaluation uniform.

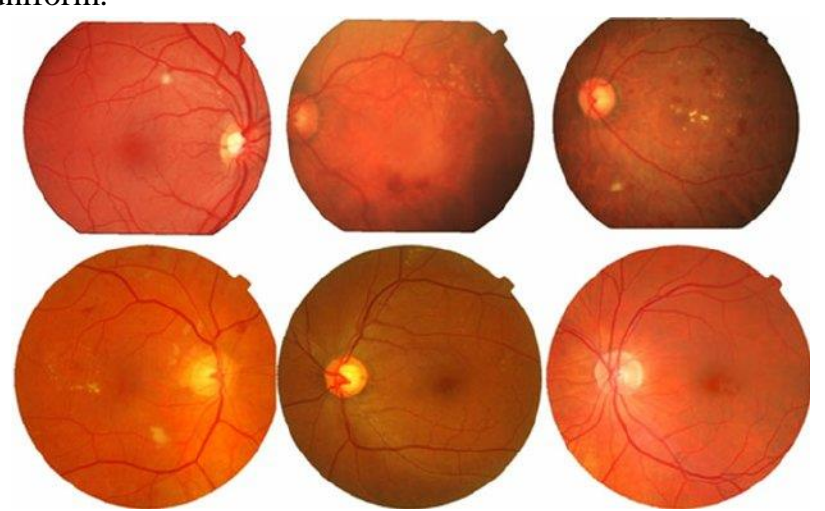

Fig. 3: Rows 1 and 2 contain Different Retinal Images from DRIVE and MESSIDOR Databases Respectively

The visible spectrum can be divided into thirds; the short, intermediate, and long wavelengths, which represent the primary colors: blue, green, and red. Red, green and blue filters each transmit one-third of white light while blocking the other two-thirds. The red filter lightens the red while darkening the others [16]. The blue and green filters have similar effects - lightening objects that are the same color as the filter while darkening the other two primary colors. To realize this, the input color image [Fig. 4(a)] is first converted to RGB image [17]. Thus intensity is improved using the RGB technique and thereby the contrast enhancement of the input [Fig. 4(b)].

The fundus image databases used in this work have resolution and illumination variations. Often, an image is composed of some underlying ideal structure, which we want to detect and describe, together with some random noise or artifact, which we would like to remove. To reduce its impact on the algorithm, we have used Gaussian filtering method [18].

For example, a simple model is that pixels from the image region of a uniform object have value $j_{r}+N(0, \mu)$, where $j_{r}$ is some expected grey level for ideal imaging conditions and $N(0, \mu)$, is Gaussian noise of mean $O$ and standard deviation $\mu$. Gaussian noise has been added to the ideal image to create the noisy image in the center: note that the noisy values have been clipped to remain within the interval [0; 255]. Noise which varies randomly above and below a nominal brightness value for a region can be reduced by averaging a neighbourhood of values. The process uses a weighted average of an input pixel (let $r$ and $c$ ) and its neighbors to calculate an output pixel.

$$
\text { Output }[\mathrm{r}, \mathrm{c}]=\left(\sum_{i=-2}^{+2} \sum_{i=-2}^{+2} \operatorname{In}\left[\mathrm{r}+\mathrm{i}_{x} \mathrm{c}+\mathrm{j}\right] / 25\right)
$$

Equation defines a smoothing filter that averages 25 pixel values in a $5 \times 5$ neighbourhood of the input image pixel in order to create a smoothed output image [19]. Thus Gaussian filtering is basically doing smoothing, sharpening, or enhancing an edge and applying effects in the input image for contrast enhancement.

Here the errors and noises caused during the acquisition of the images are removed by above filtering and thereby enhances the local contrast of an image. This extracts the fundus image from background and enhances its quality by removing noisy areas. 


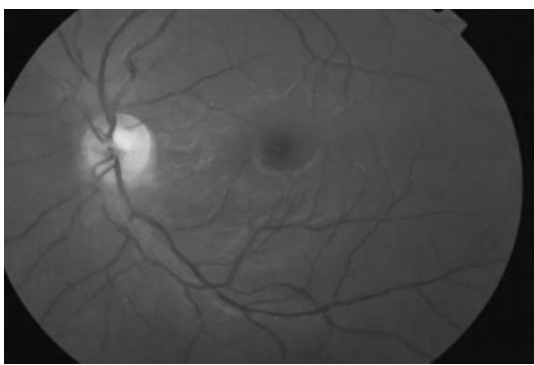

Fig. 4: Gaussian Filtered Image (Green Color Space)

\section{Optic Disc Detection and Masking}

Optic disc is a homogeneous structure of fundus image appearing bright and yellowish in color. The OD center and its diameter give information such as position of BV origin and macula region. The OD has various explicit properties that are useful for its detection. These include high intensity, circular structure and BV organization. The OD is circular and structurally uniform whereas exudates are irregular and non-uniform structures [4]. Another important feature of OD is the presence of $\mathrm{BV}$ origin and containing a network of $\mathrm{BV}$ around it. To determine the region which contains OD, the property of presence of maximum BV and circular shape is employed.

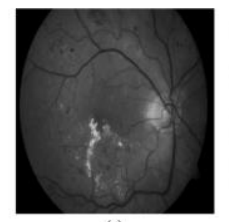

(a)
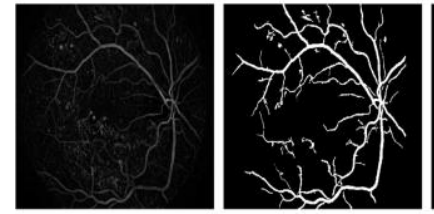
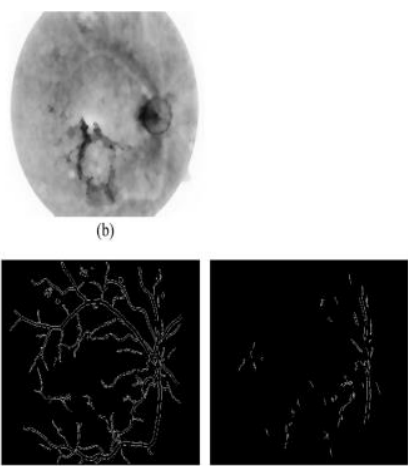

(f)
Fig. 5: Detection of BVs using GREEN Channel Image of

RGB Color Plane: (a) Green Plane of RGB; (b)

Morphological Opening Applied on Inverted Green

Plane Image; (c) OD Removed without Effecting BV; (d)

Detected BVs; (e)Edges of (d); (f) Vertical Edges of(d)

To preserve the information of BV over OD, the OD intensity is to be altered. For this purpose, the green channel image is complemented so that the BV's intensity is changed to bright and OD becomes dark [16]. On the complemented image, morphological opening is performed as given by the following equation, using a ball structuring element [4, 29]:

$$
\sigma^{(\mathrm{X})}(\mathrm{I})=\delta^{(\mathrm{X})}\left(\varepsilon^{(\mathrm{X})}(\mathrm{L})\right)
$$

Where $\delta^{(\mathrm{X})}$ represents dilation and $\varepsilon^{(\mathrm{X})}$ represents erosion applied to image ' $L$ '. The opened image is shown in Fig. 5(b). The resultant image is subtracted from the complemented green channel image, which gives removal of the OD region without affecting the BV structure in it [21] [Fig. 5(c)]. Let the image be named as 'image $X$ '. Image $X$ is then converted to binary image using Otsu's threshold [24]. The output consists of BV structure, dark lesions and noisy pixels. To isolate BVs from remaining non BV pixels, binary opening is applied to binary image, removing the pixels having less connected neighbors, according to the following equation [16] [Fig. 5(d)].

$$
f(x, y)= \begin{cases}0, \text { if } & p_{n} \leq \lambda_{1} \\ 1, & \text { if } p_{n} \geq \lambda_{1}\end{cases}
$$

Where $p_{n}$ represents the neighboring pixels and $\lambda_{1}$ represents the threshold. The value of $\lambda_{1}$ may vary from 5075 depending on image property. In the proposed algorithm three sets of values for $\lambda 1$ have been used depending on intensity of BVs. MESSIDOR and DRIVE database have less intensity BVs, hence value 55 is used. The images in HRF database contain intermediate intensity and hence value 60 is used. The BVs originate from OD and spread towards the macular direction. Generally, BV is vertically aligned in the vicinity of OD [16]. This property is used for obtaining region containing OD. From the detected BV image, the BV edges are obtained using vertical sobel mask, shown in Fig. 5(f).

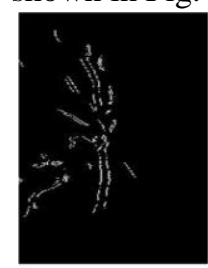

(a)

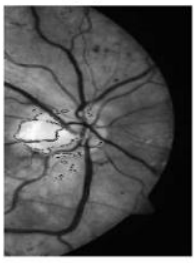

(e)

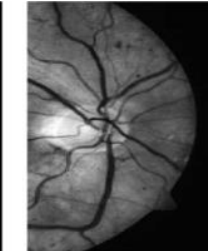

(b)

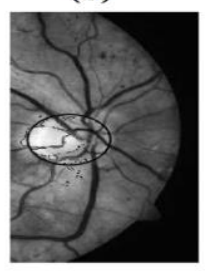

(f)

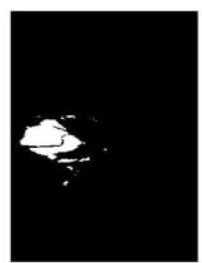

(c)

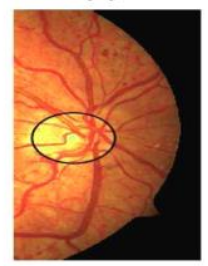

(g)

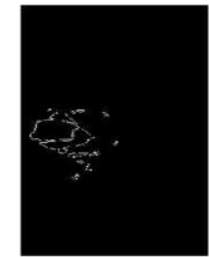

(d)

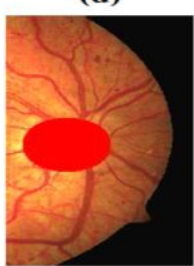

(h)
Fig. 6: OD Segmentation: (a) ROI-1 for OD, obtained from Cropped Region having Maximum Vertical BV;

(b) Corresponding ROI-1 in Gray Scale Fundus; (c) Binary Image after Applying Threshold; (d) Edges of (c); (e) Overlapped OD Edges on Gray Scale Image; (f)

Hough Transform Applied on the OD Edges; (g) Detected OD Overlapped on Colored Fundus Image; (h) OD Masked

The images of various databases have OD located either on left, right or centre. The size of OD is large enough so that even if we divide the fundus image in three vertical strips, the OD will be accommodated in any one of these strips [11]. Therefore, the image is divided vertically with a window one third of number of rows of size. Each vertical region is scanned for edge pixels and the region with maximum value is considered as region for detection of OD. Corresponding region for the processed image is shown in Fig. 6(a). After detecting this one, the corresponding gray image is considered [Fig.6 (b)]. The histogram of gray scale region is investigated for extracting the high intensity pixels representing OD. For isolating the bright pixels, Otsu's threshold [24] is used [Fig.6(c)]. Once the region of bright pixels is detected, canny edge operator is applied to find the edges of the region, shown in Fig.6 (d). To extract the distinct circular feature of OD, Hough Transform is applied to the edges [25]. The circle is later overlapped with gray scale image as shown in Fig. 6(f). The OD region coordinates are used and superimposed on the original color image [Fig. 6(g)]. Lastly, the detected OD is masked so that it does not interfere during exudates detection [26] [Fig. 6(h)]. 


\section{Detection and Analysis of Diabetic Maculopathy}

This subsection presents discussion on the method used for maculopathy detection and analysis. The presence of DM is confirmed when intensity variations (exudates, micro aneurysms and haemorrhages) are located in the neighbourhood of the macula. The variations start developing from the far neighbourhood of the macula, where there exist BVs.

But with the progression of DR, macula also gets affected [27]. The condition becomes critical when these involve fovea. The main objective therefore is to detect intensity variations near the fundus. Thereafter, the locations of the detected variations were checked in the macula neighbourhood for the analysis of maculopathy.

Subsequent to segmenting the image database, the next step of the proposed systems is the Feature extraction [27, 28]. Feature extraction is the most significant step in image classification. It helps in separating the feature of an image as ideal as possible. Feature extraction techniques are applied to get the feature that will be useful in classifying and recognizing the images.

Numerous methods are used to extract features like frequency, texture and shape as feature vector. The techniques for feature extractions are classified are shown in Fig. 1. The features are categorised on the various key component of image data like color intensity, edges of the objects present in image, texture, etc. [1].

The efficiency of feature extraction method enhances the further processing of an image to a great extent. These features can be used in image matching and retrieval. These applications require the compact and relevant information to achieve high degree of accuracy. Feature extraction technique is used to extract the features by keeping as much information as possible from large set of data of image.

Texture based feature extraction can be classified as spatial and spectral texture based on their various advantages to use in the image processing. Spatial texture is easy to use and can be extract information from any shape. These feature are very sensitive to noise and distortions. Spectral texture is robust and requires less computation.

In this work, we have used discrete wavelet transform and support vector machine classifier for automated detection of normal and diabetic maculopathy classes.

Here we use Support vector machine (SVM) as classification system [30, 31]. It is primarily a classifier method that performs classification task in a multidimensional space that separate cases of different class labels by constructing hyper planes.

Dummy variables are created for categorical variables Database. The extracted features are using for training Support Vector Machine, during this training phase we create a database of maculopathy features. After training phase the stored database is used for detection purpose.

\section{Feature Extraction Techniques}

Feature extraction plays an important role in Image detection system and better selection of feature gives higher accuracy. It separates the visual information from the image and stores them in the form of feature vectors in a feature database.

These feature vectors are used to compare the query image with the images stored in the database. In the field of classification, features can be characterized as a way to distinguish one class of object from another. The three main approaches used for the categorization are based on the Frequency, shape and texture. These feature extraction techniques are as follows:

\section{Shape Features Extraction}

Shape features are mostly used for finding and matching shapes, recognizing objects or making measurement of shapes. These methods involve two type of approach. First is continuous approach which does not divide shape into subpart. It uses the integral boundary to derive the feature vector. Second is Discrete (Global) Approach divides the shape boundary into sub part and compute the multidimensional feature vector.

The shape of an object is determined by its external boundary abstracting from other properties such as color, content and material composition, as well as from the object's other spatial properties. Area, orientation, major axis, minor axis and eccentricity are some of the characteristics used for shape features extraction technique here [32].

\section{- Area and Orientation}

The area is the number of pixels in a shape. The convex area of an object is the area of the convex hull that encloses the object. The Orientation is the overall direction of the shape or the angle between horizontal axis and major axis.

\section{- Minor Axis}

The minor axis is the $(\mathrm{x}, \mathrm{y})$ endpoints of the longest line that can be drawn through the object whilst remaining perpendicular with the major-axis. The minor axis endpoints $(\mathrm{x} 1, \mathrm{y} 1)$ and $(\mathrm{x} 2, \mathrm{y} 2)$ are found by computing the pixel distance between the two border pixel endpoints. The result is measure of object width.

The minor-axis length of an object is the pixel distance between the minor-axis endpoints and is given by the relation $[32,33]$ :

$$
\text { minor axis length }=\sqrt{(x 2-x 1)^{2}+\left(y^{2}-y 1\right)^{2}}
$$

\section{- Major Axis}

The major axis is the $(\mathrm{x}, \mathrm{y})$ endpoints of the longest line that can be drawn through the object. The major axis endpoints (x1, y1) and (x2, y2) are found by computing the pixel distance between every combination of border pixels in the object boundary and finding the pair with the maximum length.

The result is measure of object length. The major-axis length of an object is also the pixel distance between the major-axis endpoints and is given by the relation:

major axis length $=\sqrt{(x 2-x 1)^{2}+(y 2-y 1)^{2}}$

- Eccentricity

It is the ratio of the length of the short (minor) axis to the length of the long (major) axis of an object:

$$
\text { Eccentricity }=\frac{\text { axislength }}{\text { axislength }_{\text {long }}}
$$

The result is a measure of object eccentricity, given as a value between 0 and 1 . Sometimes it is known as ellipticity [33].

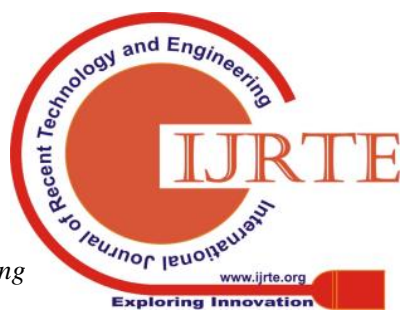




\section{Texture Features Extraction}

Texture is a very important characteristic for the analysis of medical images [34]. Texture synthesis is a common technique to create large textures from usually small texture samples, for the use of texture mapping in surface. Texture classification produces a classified output of the input image where each texture region is identified with the texture class it belongs.

The textural features based on gray-tone spatial dependencies have a general applicability in image classification. Gray level co-occurrence matrix (GLCM) has proven to be a powerful basis for use in texture classification. It is defined as "A two dimensional histogram of gray levels for a pair of pixels, which are separated by a fixed spatial relationship." [35].

This presents an application of GLCM to extract second order statistical texture features. Given an image composed of pixels each with an intensity (a specific gray level), the GLCM is a tabulation of how often different combinations of gray levels co-occur in an image or image section. Texture feature calculations use the contents of the GLCM to give a measure of the variation in intensity at the pixel of interest. Few of the common statistics like Contrast, Entropy, Energy and Homogeneity applied to co-occurrence probabilities are discussed ahead.

\section{- Contrast:}

$$
\text { Contrast }=\sum_{i=0}^{N-1} \sum_{j=0}^{N-1}(i-j)^{2} M\left(i_{v} j\right)
$$

The contrast (Con) is defined in Equation (4.14), is a measure of intensity of a pixel and its neighbor over the image. In the visual perception of the real world, contrast is determined by the difference in the color and brightness of the object and other objects within the same field of view.

\section{- Entropy:}

This statistic measures the disorder or complexity of an image.

$$
\text { Entropy }=-\Sigma_{i} \sum_{j} g_{i j} \log _{2} g_{i j}
$$

The entropy is large when the image is not texturally uniform and many GLCM elements have very small values. Complex textures tend to have high entropy. Entropy is strongly, but inversely correlated to energy [37, 38].

\section{- Energy:}

$$
\text { Energy }=\Sigma_{i} \Sigma_{j} g_{i j}^{2}
$$

It measures the textural uniformity that is pixel pair repetitions. It detects disorders in textures. Energy reaches a maximum value equal to one. High energy values occur when the gray level distribution has a constant or periodic form. Energy has a normalized range. The GLCM of less homogeneous image will have large number of small entries [35, 36, and 37].

\section{- Homogeneity:}

$$
\text { Homogeneity }=\Sigma_{i} \Sigma_{j} \frac{1}{1+(i-j)^{2}} g_{i j}
$$

It measures image homogeneity as it assumes larger values for smaller gray tone differences in pair elements. It is more sensitive to the presence of near diagonal elements in the GLCM. It has maximum value when all elements in the image are same.

GLCM contrast and homogeneity are strongly, but inversely, correlated in terms of equivalent distribution in the pixel pairs population $[36,37,38]$.

\section{Discrete Wavelet Transform Feature Extraction}

Here the Wavelet concept facilitates feature extraction. Discrete wavelet transform is used here to extract characteristics from a signal on various scales proceeding by successive high pass and low pass filtering. The wavelet coefficients are the successive continuation of the approximation and detail coefficients [39]. Using twodimensional DWT an image is divided into four sub-bands namely LL which has coarse information and the other LH, HL and LL have detailed information as shown in the below figure7. The coarse information is an approximation image which is used for next level of resolution to decompose and other three are horizontal, vertical and diagonal wavelet features and are detailed coefficients of sub-bands which are generated when decomposed.

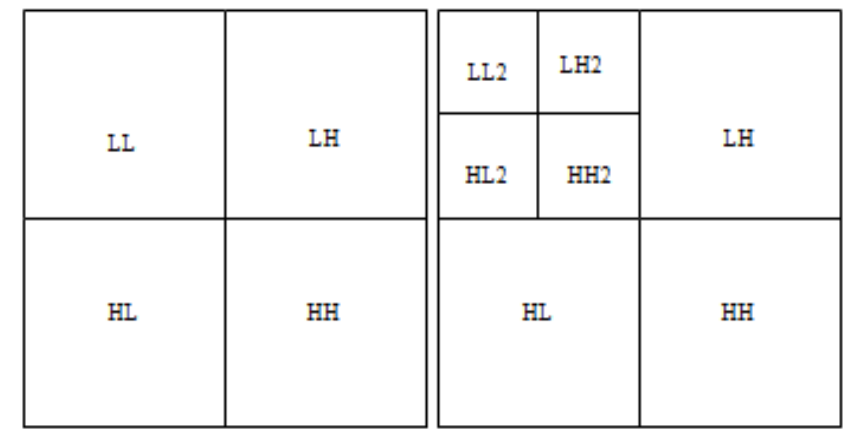

Fig. 7: First-level and Second-level of Decomposition of DWT

It is performed on rows first then on columns, $\mathrm{H}$ and $\mathrm{L}$ denote high-pass and low-pass channels respectively and for decomposition the size of an image is reduced to half. For example, if the sub-band image is produced using low pass filter on columns and high pass filters on rows, its LH subband and LL is an approximation image, LH deals with vertical, HL deals with horizontal and $\mathrm{HH}$ deals with diagonal features [ 39 and 40].

For all the $\mathrm{N}$ blocks the values are stored in the variable, which is a feature matrix and where array has all the values of all the blocks and each block consists of values which contains coordinates of row and column and along with this values of sum of all horizontal, vertical and diagonal features of each block, which is computed using summation of all horizontal values to the size of corresponding resolution and horizontal coefficient of wavelet transform is denoted by $\mathrm{H}$, vertical by $\mathrm{V}$ and $\mathrm{D}$ by diagonal for each block of an image, where $\mathrm{H}$ focuses on horizontal edge features, $\mathrm{V}$ focuses on vertical edge features and $\mathrm{D}$ focuses on diagonal edge features and these have the detailed information which are produced when applied on every resolution and also produce an approximate image which has rough data and used to apply DWT for every resolution and this gives four sub band images when performed on rows first and then on columns. These features are used to detect the correct text blocks of images and are fed for classification stage.

Daubechies wavelet is the most commonly used set of discrete wavelet transforms [30]. Daubechies orthogonal 
Wavelets db1-db10 are commonly used. The index number refers to the number $\mathrm{N}$ of coefficients. The following statistical textural features such as mean, moment, variance, standard deviation, entropy, skewness and Kurtosis information measures are extracted from each gray level cooccurrence matrix and take the average of all the features extracted from the four gray level co-occurrence matrices.

The mean is obtained by taking the average of all the components of the space used. Moment is usually called homogeneity that measures the local homogeneity of an image. Variance is a measure of heterogeneity. Standard deviation is another statistical measure used and is defined as a quantity that expresses how much the members of a channel differ from the mean value for the channel. Entropy is the measure of image information content, which can be interrupted as the average uncertainty of the information source. Skewness measures the degree and direction of symmetry or asymmetry of the distribution. Kurtosis measures how peaked a distribution is and the lightness or heaviness of the tails of the distribution [41, 42, 43]. It measures how much of the distribution is actually located in the tails.

\section{Support Vector Machine (SVM) Classification Technique}

Using a nonlinear mapping to get a higher dimension data from original training is done by support vector machine (SVM) algorithm With-in new measurement, it examinations for linear optimal extrication hyper-plane. A hyper-plane is a "judgment border" unscrambling the types of unique class after another. Through applicable non-linear planning to a satisfactorily high measurement, data as of two modules can continuously be disconnected by a hyper-plane. The SVM discover this hyper-plane by means of supportvectors and margins. An SVM model can be considered as points in space that can be categorized in to different categories. Here we use the extracted features for training Support Vector Machine, during this training phase we create a database of affected images. After training phase the stored database is used for detection purpose.

In SVM the decision boundary should be as far away from the data of both classes as possible. Let us consider that we have data points belonging to two classes, $\mathrm{A}+$ and A-. Each point in the dataset comes with a class label $\mathrm{y},+1$ or 1 , indicating one of two classes $\mathrm{A}+$ and $\mathrm{A}-$. Let us start with a strictly linearly separable case, i.e. there exists a hyper plane which can separate the data A+ and A-. In this case we can separate the two classes by a pair of parallel bounding planes (eq.4, 5) [5].

$$
\begin{aligned}
& w^{T} x+b=+1 \\
& w^{T} x+b=-1
\end{aligned}
$$

Where $w$ is the normal vector to these planes and $b$ determines their location relative to the origin. The first plane of (eq. 2) bounds the class $A+$ and the second plane bounds the class $A$-. SVM achieves better prediction ability via maximizing the margin between two bounding planes. Hence, SVM searches for a separating hyper plane by maximizing $\frac{2}{\|w\|_{2}}$. Hence the linear separating hyper plane is the plane,

$$
w^{T} x+b=0
$$

By maximizing the margin between the bounding planes

we get an optimal solution $\left(w^{*}, b^{*}\right)$. The data points on the bounding planes $w^{* T} x+b^{*}= \pm 1$ are called support vectors. Once we have the training result, all we need to keep in our databases are the support vectors. If the classes are linearly inseparable then the two planes bound the two classes with a soft margin determined by a nonnegative slack vector variable $\xi$, that is:

$$
\begin{gathered}
w^{* T} X_{\mathrm{i}}+b^{*}+\xi_{\mathrm{i}} \geq+1 \text { for } X_{\mathrm{i}}^{T} \in \mathrm{A}_{+} \\
w^{* \mathrm{~T}} \mathrm{X}_{\mathrm{i}}+\mathrm{b}^{*}-\xi_{\mathrm{i}} \leq-1 \text { for } \mathrm{X}_{\mathrm{i}}^{\mathrm{T}} \in \mathrm{A}_{-}
\end{gathered}
$$

Many datasets cannot be well separated, even after using a soft margin, by a linear separating hyper plane, but could be linearly separated if mapped into a higher or much higher dimensional space by using a nonlinear map rather than mapping individual features to higher dimension. Kernel method is used for this purpose. The kernel computes the dot product which would otherwise be much more expensive to compute explicitly $[30,31]$.

\section{Healthy vs. DM Classification}

For evaluating the proposed algorithm, three publicly available fundus image databases are used: the DRIVE (Digital Retinal Images for Vessel Extraction) [12], MESSIDOR (Methods to evaluate segmentation and indexing techniques in the field of retinal ophthalmology) [14] and HRF (High-Resolution Fundus Image) [15]. The method is evaluated in 332 images using these three databases. The intensity variations are quantitatively evaluated by comparing with manually labeled image. The comparison is based on number of abnormality pixels detected. For a given infection condition, the finest conceivable test can be chosen in view of three characteristics. They are Sensitivity (SS), Specificity (SF) and Accuracy. How great the test is at identifying a positive infection is evaluated by sensitivity. How likely the patients

\begin{tabular}{|c|c|c|c|c|c|}
\hline \multirow{2}{*}{ Databases } & \multirow{2}{*}{$\begin{array}{l}\text { Images } \\
\text { tested }\end{array}$} & \multicolumn{2}{|c|}{ Maculopathy cases } & \multicolumn{2}{|c|}{$\begin{array}{l}\text { Analyzing } \\
\text { parameter }\end{array}$} \\
\hline & & $\begin{array}{l}\text { Positive } \\
\text { cases }\end{array}$ & $\begin{array}{l}\text { Detected } \\
\text { cases }\end{array}$ & $\begin{array}{l}\text { SS } \\
(\%)\end{array}$ & $\mathrm{SF}(\%)$ \\
\hline DRIVE & 10 & 9 & 9 & 100 & 100 \\
\hline MESSIDOR & 310 & 305 & 295 & 96.72 & 96.45 \\
\hline HRF & 12 & 11 & 11 & 100 & 100 \\
\hline \multicolumn{4}{|c|}{ Average Value } & 98.91 & 98.82 \\
\hline
\end{tabular}
without sickness can be effectively eliminated is done with the help of specificity. Accuracy measures rightness of the test.

Table I: Summary of Maculopathy Detection

The performance of the proposed algorithm is assessed based on particular characteristics (Table I and II). This can be summarized in a graph plot between specificity and

\begin{tabular}{|c|c|c|c|c|}
\hline \multirow[b]{2}{*}{ Databases } & \multirow{2}{*}{$\begin{array}{l}\text { No. of } \\
\text { image tested }\end{array}$} & \multicolumn{2}{|c|}{ Analyzing parameter } & \multirow{2}{*}{$\begin{array}{l}\text { Accuracy } \\
(\%)\end{array}$} \\
\hline & & SS (\%) & SF (\%) & \\
\hline DRIVE & 10 & 100 & 100 & 100 \\
\hline MESSIDOR & 310 & 96.72 & 96.45 & 96.58 \\
\hline HRF & 12 & 100 & 100 & 100 \\
\hline \multicolumn{2}{|c|}{ Average Value } & 98.91 & 98.82 & 98.86 \\
\hline
\end{tabular}
sensitivity (fig. 8), that are used to determinate how well a test can discriminate between patients who have the disease and those who do not.

Table II: Summary of Detection by Accuracy 
The following terms are used along with the description of sensitivity, specificity. They are true positive (TP), true negative (TN), false negative (FN), and false positive (FP). If a disease is proven present in a patient, the given diagnostic test also indicates the presence of disease, the result of the diagnostic test is considered true positive. Similarly, if a disease is proven absent in a patient, the diagnostic test suggests the disease is absent as well, the test result is true negative (TN). Both true positive and true negative suggest a consistent result between the diagnostic test and the proven condition. If the diagnostic test indicates the presence of disease in a patient who actually has no such disease, the test result is false positive (FP). Similarly, if the result of the diagnosis test suggests that the disease is absent for a patient with disease for sure, the test result is false negative (FN). Both false positive and false negative indicate that the test results are opposite to the actual condition. Here Sensitivity and specificity are described in terms of TP, TN, FN and FP. So the sensitivity and specificity measurements are obtained as follows [40, 41]:

$$
\begin{aligned}
& \text { Sensitivity }(S S)=\frac{T P}{T P+F N} \\
& \text { Specificity }(S F)=\frac{T N}{T N+F P}
\end{aligned}
$$

The sensitivity is the measurement of actual variations of pixels. More the sensitivity better is the detection. The Specificity represents the proportion of non-varying pixels, which are correctly rejected. The intensity varying pixels are very few compared to non-intensity varying pixels.

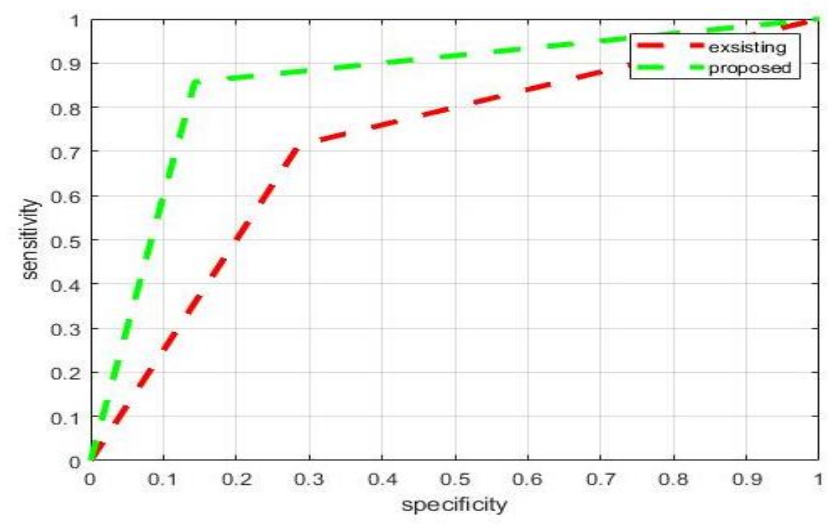

Fig. 8: Sensitivity vs. Specificity for Existing/Proposed Methods

To measure the performance, accuracy was employed with the average of all three observations recorded as the final classification rate [42].

$$
\text { Accuracy }=\frac{5 S+S F}{2}
$$
cases. This result can be seen in Tables I-III. The highest average accuracy of $98.86 \%$ (refer to Table II) was accomplished using DWT with SVM, achieving a sensitivity of $98.91 \%$ and specificity of $98.82 \%$.

The performance of proposed algorithm in comparison with Deepak and Sivaswamy [44], Siddalingaswamy and Prabhu [45], Tariq et al. [46] and Zaidi et al. [47] is given in Table III. Result shows that the algorithm performs better in detecting the DM on various images. The numerical results were obtained on the funds image database comprised of 332 images divided into 7 Healthy and 325 DM samples. The DRIVE database contains 10 training set and 10 test set of images out of which 9 images have signs of DM.
It is noted that SVM achieved better performance in all

MESSIDOR database has 305 images with different resolutions of $1440 \times 960,2240 \times 1488$ and $2304 \times 1536$ pixels, have signs of DM. HRF database consists of 12 images with $3504 \times 2336$ pixels. Out of 12 images, 11 have signs of DM and 1 image has sign of glaucoma.

Table III: Comparison of Diabetic Maculopathy Detection

\begin{tabular}{|l|l|l|}
\hline Methods & $\begin{array}{l}\text { Average } \\
\text { sensitivity (\%) }\end{array}$ & $\begin{array}{l}\text { Average } \\
\text { specificity (\%) }\end{array}$ \\
\hline $\begin{array}{l}\text { Deepak and Sivaswamy } \\
\text { [44] }\end{array}$ & 95 & 90 \\
\hline $\begin{array}{l}\text { Siddalingaswamy and } \\
\text { Prabhu [45] }\end{array}$ & 95.6 & 96.15 \\
\hline Tariq et al. [46] & 96.7 & 98.29 \\
\hline Zaidi et al. [47] & 93.5 & 95.81 \\
\hline Proposed method & $\mathbf{9 8 . 9 1}$ & $\mathbf{9 8 . 8 2}$ \\
\hline
\end{tabular}

The detection of DM is better as compared to other existing work with an accuracy of $98.86 \%$. The maculopathy assessment is done on all the images containing abrasion. The manual identification done earlier has been used as gold standard or comparing the result. The result shows that the algorithm performs better for DM identification indifferent types of images.

\section{CONCLUSION}

Long-drawn-out diabetes causes severe damage to the vision through leakage of blood and blood constituents over the retina. The effect of the leakage becomes more threatening when these abnormalities involve the macula. This method overcomes the problems of failures in fovea and macula detection and the results of proposed techniques are better as compared to other algorithms. The improved detection rate of fovea enhances the maculopathy assessment rate to a higher rank. To the best of our knowledge, there is very little publication work has happened to detect DM using retinal images. The algorithm is advantageous as emphasis has been given to all the stages of the analysis and it's a tool for automatic inspection of diabetic maculopathy. These techniques distinguish Healthy and DM classes with an average accuracy of $98.86 \%$, and we believe our method can be helpful in reducing the cost and simplifying the procedure of diagnosis and help to develop new therapies for diabetic maculopathy.

\section{REFERENCES}

1. World health Organization 〈https://www.who.int/newsroom/fact-sheets/detail/diabetes $\rangle$,October 2018.

2. International Diabetes Federation (IDF) s Diabetes Atlas 〈http://www.retinaindia.org/retinal-disorders/diabeticretinopathy, May 2015.

3. R. O. Duncan et al., "Retinotopic organization of primary visual cortex in glaucoma: A method for comparing cortical function damage to the optic disk," Invest. Ophthalmol. Sci., vol. 48, pp. 733-744, Feb. 2007.

4. Jyoti Prakash Medhi, Samarendra Dandapat "An effective fovea detection and automatic assessment of diabetic maculopathy in color fundus images", Computers in Biology and Medicine 74 (2016) 30-44. 
5. Sisodia, D., Shrivastava S.K., Jain R.C., SVM for face recognition. Proceedings-2010 International Conference on Computational Intelligence and Communication Networks, CICN2010, 554-559.

6. Raman R.; Gella L.; Srinivasan S.; Sharma T., Diabetic retinopathy: An epidemic at home and around the world. Indian J. Ophthalmol. 2016, 64, 69-75. [CrossRef] [PubMed].

7. A. Augustin, F. Bandello, G. Coscas, C.C. Garcher, et al., Macular Edema. A Practical Approach, Karger, 2010.

8. Reichel, E.; Salz, D. Diabetic retinopathy screening. In Managing Diabetic Eye Disease in Clinical Practice; Springer: Berlin, Germany, 2015; pp. 25-38.

9. Yanoff, Myron (2009). Ocular Pathology. Elsevier Health Sciences. p. 393. ISBN 0-323-04232-5.

10. Remington, Lee Ann (29 July 2011). Clinical Anatomy of the Visual System. Elsevier Health Sciences. pp. 314-5. ISBN 14557-2777-6.

11. International Diabetes Federation (IDF). IDF Diabetes Atlas; IDF: Brussels, Belgium, 2017.

12. DRIVE Database /http://www.isi.uu.nl/ research/ databases/ drive), May 2017.

13. DIARETDB1 Database 〈http: //www2. it.lut.fi/ project/ imageret/ diaretdb1/>, May 2017.

14. MESSIDOR Database 〈http://messidor.crihan.fr/downloaden.php , May 2017.

15. High-Resolution Fundus (HRF) Image Database 〈http://www5.cs.fau.de/re search/data/fundus-images/〉, May 2017.

16. Ciulla, T.A.; Amador, A.G.; Zinman, B. Diabetic retinopathy and diabetic macular edema: Pathophysiology, screening, and novel therapies. Diabetes Care 2003, 26, 2653-2664. [CrossRef] [PubMed].

17. D. Michael, M. Niemeijer, A. Osareh, D. Simmons, et al., Automated Image Detection of Retinal Pathology, CRC Press, Taylor \& Francis Group, 2010.

18. Choomchuay, Somsak, and Keokanlaya Sihalath. "An application of second derivative of gaussian filters in fingerprint image enhancement." Bioinformatics and Biomedical Engineering (iCBBE), 2010 4th International Conference on. IEEE, 2010.

19. Baek, Jongmin, and David E. Jacobs. "Accelerating spatially varying Gaussian filters." ACM Transactions on Graphics (TOG). Vol. 29. No. 6. ACM, 2010.

20. H. Li, O. Chutatape, Automated feature extraction in color retinal images by a model based approach, IEEE Trans. Biomed. Eng. 51(2) (2004)246-254.

21. Bandello, F.; Parodi, M.B.; Lanzetta, P.; Loewenstein, A.; Massin, P.; Menchini, F.; Veritti, D. Diabetic macular edema. In Macular Edema; Karger Publishers: Basel, Switzerland, 2010; Volume 47, pp. 73-110.

22. J. Welfer, D. Scharcanski, D.R. Marinho, A coarse-to-fine strategy for auto-matically detecting exudates in color eye fundus images, Comput. Med. Ima-ging Graph. 34 (3) (2010)228-235.

23. J.M. Provis, P.L. Penfold, E.E. Cornish, T.M. Sandercoe, M.C. Madigan, Anatomy and development of the macula: specialization and the vulnerability to macular degeneration, Clin. Exp. Optom. Pubmed 88(5) (2005)269-281.

24. N. Otsu, "A threshold selection method from gray-level histograms", IEEE Trans. Syst. Man Cybern. 9(1) (1979).

25. S. Sekhar, W. Al-Nuaimy, A. Nandi, Automated localisation of optic disk and fovea in retinal fundus images, in: European Signal Processing Conference, Switzerland, 2008.

26. P.F. Sharp, J. Olson, F. Strachan, J. Hipwell, A. Ludbrook, et al., The Value of Digital Imaging in Diabetic Retinopathy, Gray Publishing, Tunbridge Wells, Kent,2003.

27. L. Giancardo, F. Meriaudeau, T.P. Karnowski, H. Li, S. Garg, K.W. TobinJr, E. Chaum, Exudate-based diabetic macular edema detection in fundus images using publicly available datasets, Med. Image Anal.16 (1) (2012) 216-226.
28. R. C. Gonzalez and R. E. Woods, Digital Image Processing. NJ: Prentice Hall, 2001

29. P. Soille, "Morphological Image Analysis: Principles and Applications", 2nd edition, Springer, Heidelberg, 2003.

30. K. Kunadumrongrath and A. Ngaopitakkul, Member, IAENG "Discrete Wavelet Transform and Support Vector Machines Algorithm for Classification of Fault Types on Transmission Line"

31. C. Kose, U. Åevik, O.Genaliolu, "Automatic segmentation of age-related macular degeneration in retinal fundus images", Comput. Biol. Med. 38 (5) (2008) 611-619.

32. Linde Oskar, Lindeberg Tony, "Composed Complex-Cue Histograms: An Investigation of the Information Content in Receptive Field Based Image Descriptors for Object Recognition", In Computer Vision and Image Understanding 116 (2012) 538\{5602.DOI: 10.1016/j.cviu.2011.12.03, March-16, 2012.

33. Wu Jun, Xiao Zhitao, "Video Surveillance Object Recognition Based on Shape and Color Features", 3rd International Congress on Image and Signal Processing (CISP2010), 2010.

34. A. Arivazhagan and L. Ganesan, "Texture classification using wavelet transform," Pattern Recog. Lett., vol. 24, pp. 15131521, 2003.

35. R. M. Haralick, K. Shanmugam and I. Dinstein "Textural features for Image Classification", IEEE Transactions on Systems, Man and Cybernetics, Vol.3, pp. 610-621, November 1973.

36. R. N. Sutton and E. L. Hall, "Texture measurement for automatic classification of pulmonary disease", IEEE Trans. Compute., vol. C-21, (1972) July, pp. 667-676.

37. S. Mori Tamura and T. Yamawaki, "Textural Features Corresponding to Visual Perception", IEEE Transactions on Systems, Man and Cybernetics, vol. smc-8, no. 6, (1978) June, pp. 460-473.

38. K. R. M. Haralick, "Textural Features for Image Classification", IEEE Transactions on Systems, Man and Cybernetics, vol. smc-3, no. 6, (1973) November, pp. 610621.

39. K. Huang and S. Aviyente, "Wavelet feature selection for image classification," IEEE Trans. Image Process., vol. 17, no. 9, pp. 1709-1720, Sep. 2008.

40. Daubechies, Ten Lectures on Wavelets. Philadelphia, PA: Society for Industrial and Applied Mathematics, 1992.

41. Roopashree. C. S. 1, Saritha Chakrasali "Detection of Diabetic Retinopathy using Wavelet Transform and SVM Classifier", International Journal for Research in Applied Science \& Engineering Technology (IJRASET).

42. "Detector Performance Analysis Using ROC Curves MATLAB \& Simulink Example". www.mathworks.com. Retrieved 11 August 2016.

43. https://en.wikipedia.org/wiki/Sensitivity_and_specificity.

44. K.S. Deepak, J. Sivaswamy, Automatic assessment of macular edema from color.

45. P.C. Siddalingaswamy, K. Prabhu, Automatic grading of diabetic maculopathy.

46. A. Tariq, M.U. Akram, A. Shaukat, S.A. Khan, A computer aided system for grading of maculopathy, in: Cairo International Biomedical Engineering Conference (CIBEC), 2012, pp. 31-3.

47. Z.Y. Zaidi, M.U. Akram, A. Tariq, Retinal image analysis for diagnosis of macular edema using digital fundus images, in: IEEE Jordan Conference on Applied Electrical Engineering and Computing Technologies (AEECT), 2013. 